\title{
23
}

\section{Experiences with Groupware Development under CORBA}

\author{
Thilo Horstmann and Markus Wasserschaff \\ National Research Centre for Informatics and Information Technology (GMD), \\ CSCW Research Group, Postfach 1316, D-53731 St. Augustin, Germany
}

This paper summarizes our experiences of groupware application development based on the new CORBA technology. We designed and developed a system that implements the metaphor of circulation folders on computer systems. Like its real-world counterpart, an electronic circulation folder can contain any kind of object, e. g. documents, annotations, spreadsheets, or graphics. When a user has finished his work with a circulation folder it will automatically be sent to the next user according to an user defined control tag. The application is built upon the new Distributed Smalltalk, Version 2.1 of Hewlett-Packard. HPDST is an application framework for building distributed, cooperative applications. It provides a full implementation of the CORBA 1.1. standard and extensions that have been submitted to OMG for CORBA 2.0. Additionally, there are class libraries according to Hewlett-Packard's Distributed Application Architecture (DAA) which particularly simplify the implementation of groupware applications.

Keyword Codes: C.2.4, D.1.5, H.4.1

Keywords: CORBA, Smalltalk, CSCW, Groupware

\section{INTRODUCTION}

The CSCW group of the Institute of Applied Information Technology at the German Research Centre of Computer Science (GMD-FIT.CSCW) has been developing groupware applications for over 10 years. The institute's research was mainly focused on developing work flow and task management systems (Domino [5], The Task Manager [6]), e-mail systems, video conferencing tools and organization information systems [8]. Currently the research group is involved in the Politeam project which is intended to support the coordination of work in German Ministries. It was set up because of the decision of the German Bundestag to move main parts of the government and ministries from Bonn to Berlin [4].

Successful groupware development currently requires consideration of many different areas of computing, such as distributed computing, (data sharing and migration across networks, data consistency), database technology (long lasting transactions, data persistency), user interface design (multi-user interfaces, adaptable interfaces), and others. Although most of these points are not an immediate research subject to $\mathrm{CSCW}$ they require much conceptual and implementational effort during the groupware development cycle. 
In particular, we were forced to focus much of our intention on the network layer of our applications. Although we designed and implemented our applications in an object-oriented fashion we have had to implement the communication level based on low-level protocols, e.g. TCP/IP, ISO/DE or RPC. This has been due to the fact that were no industrial-grade tools available for cross-platform, object-oriented programming.

The implementation effort of the network layer had often influenced the design process of our groupware applications. In some cases, we were even forced to drop important functional requirements to the application because the expected implementational effort would have exceeded our resources. ${ }^{1}$

To keep the overall design of the network layers simple, we have often chosen to provide the servers and clients with a functional interface, although this is contradictionary to the object-oriented programming paradigm.

As a result, one of the most important issues in CSCW, awareness, notification and eventing, was often implemented in a very rudimentary way. The classical (functional) client server approach does not allow the server to actively inform its clients of data changes. Instead, the server must first generate a representation of the changed data, which can be accessed by the clients by means of polling mechanisms. In turn, clients must filter and interpret the server's data to get the changes relevant for the corresponding user.

The classical client-server approach has turned out to be unacceptable for supporting many cooperative activities. In groupware applications supporting loosely coupled cooperation, in which changes occur rarely, the permanent client polling implies a disproportional load of both the network, and the clients' machines. This holds particularly in wide area networks in which the clients might be separated by long distances from the server. In tightly coupled systems, the generation and interpretation of data strongly decrease the server and client performance. These disadvantages become even more significant in applications in which cooperation changes from loosely to tightly coupled over time.

For these reasons our institute has made an evaluation of Distributed Smalltalk from Hewlett-Packard (HPDST). HPDST is a complete Smalltalk development environment based on VisualWorks from ParcPlace, Inc. extended with class libraries for distributed programming according to the CORBA 1.1 standard. Applications developed in HPDST will run without modifications to the source or binary code on all platforms supported by VisualWorks. In addition to the CORBA compliant class libraries, HPDST provides a framework for building cooperative applications. This framework includes libraries for building applications according to HP's Distributed Application Architecture (DAA) and libraries supporting the development of cooperative user interfaces.

In this paper we will first describe the functionality of the circulation folder application. After describing major concepts of the HPDST system in Section 3 and 4, we will present some details of our implementation. Finally, Section 6 analyses the experiences we made with the software development.

1. Grudin even argued that the lack of a common infrastructure has contributed to the failure of most existing cooperative systems to effectively support the work for which they were designed [2], [3]. 


\section{THE EVALUATION APPLICATION}

The HPDST application framework provides many promising features for the development of distributed, cooperative applications. To get an impression of the usefulness of these features, our institute decided to implement a typical groupware application based on HPDST. We developed a system that simulates circulation folders which are in common use in different kinds of organizations. It allows users to send arbitrary objects like documents, annotations, graphics, spreadsheets etc. to other users in a wide area network. An electronic circulation folder is routed across the net according to a user defined control tag. Access rights specify which users are allowed to modify the control tag and the contents of a circulation folder. A user should be kept informed about the state and current position of his circulation folder.

Fig. 1 shows the client start-up window. Once a user has successfully connected to the server he can access all circulation folders that have newly arrived ("In Tray"). To create and send new circulation folders, he has to open his personal workspace by double clicking on the workspace icon.

Fig. 2 shows a circulation folder which is ready to send to other users. The upper

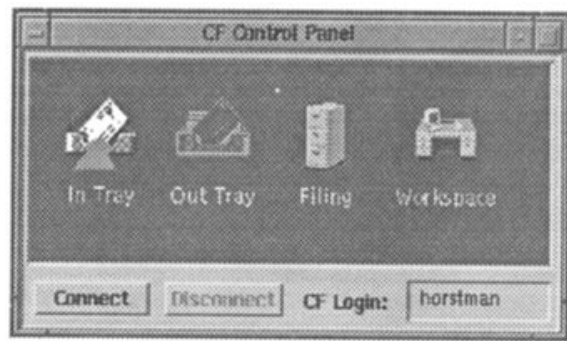

Fig. 1 Circulation Folder Login Window

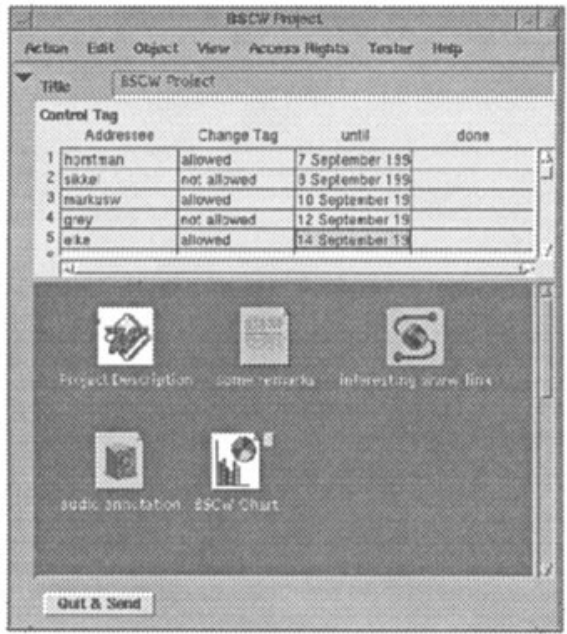

Fig. 2 Typical Circulation Folder

part of the circulation folder represents its control tag. The creator stands in the first line followed by the recipients. The creator defines who is allowed to modify the control tag and the deadline each recipient should forward the folder. Similar, the creator specifies who is allowed to delete or add objects to the folder ("Access Rights").

The circulation folder may contain external objects, e.g. a standard Microsoft Word document. If the receiver of this folder clicks on this Icon the appropriate external application will be started with this file as argument. The application to 
start is user defined and does not necessarily have to be the same as the one with the file was created. This allows the use of documents of external applications platform independently. E.g. a WinWord document might be processed on Macintosh with MacWord and on Unix with FrameMaker.

To integrate the huge amount of information resources available in the World Wide Web, users can include Uniform Resource Locators (URLs) in a circulation folder. Clicking on this Icon starts Mosaic (or a different WWW-Browser) with this URL. Since a URL can point to files created by arbitrary applications, URL's provide another way to integrate external applications. E.g. if the URL points to a postscript file, clicking on the WWW-icon would start Mosaic which in turn starts a postscript viewer with the file depicted by the URL. This approach is more generic but require to start both Mosaic and the external application.

The other objects shown in the folder are objects shipped with the HPDST framework. These are a simple text object which can be used to include personal remarks concerning the folder, a graphic object showing for instance a business chart and an audio annotation.

A circulation folder is sent by either pressing the "Quit \& Exit" button or by dragging the circulation folder icon onto the "Out Tray" icon.

When a circulation folder is sent the system creates a circulation folder adapter in the user's "Out Tray." The adapter contains the current position of the folder and is automatically updated each time the folder is sent to the next user.

Before we explain the implementation of the application in more detail, we give an overview of the HPDST system. Because of the numerous features of HPDST we will focus on those parts that mainly influenced our implementation.

\section{BRIEF OVERVIEW OF HPDST}

HPDST extends the VisualWorks System with class libraries for distributed, object-oriented programming according to the CORBA 1.1 standard [7]. This includes a portable implementation of the Object Request Broker (ORB) and Object Services. Fig. 3 shows the architecture of the system. Because HPDST does

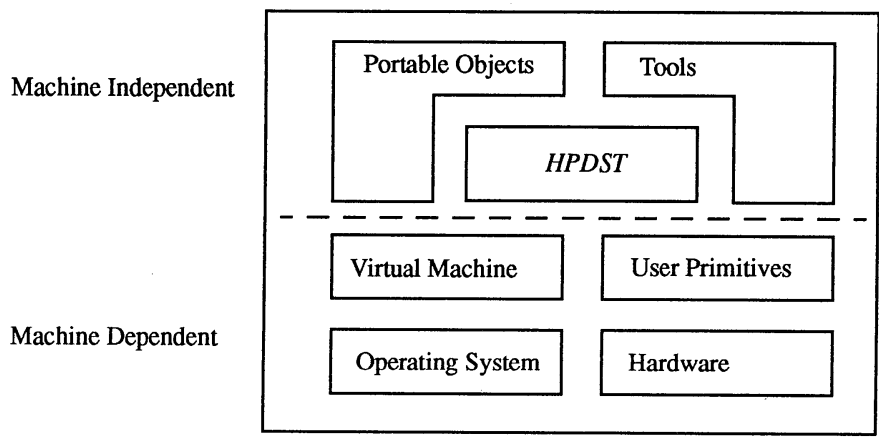

Fig. 3 HPDST Architecture. 
not modify the hardware dependent parts of the VisualWorks Smalltalk system, it runs on all platforms supported by VisualWorks. The current implementation of HPDST relies on TCP/IP communication protocols. Support of OSF DCE RPC is planned for future versions.

With HPDST, distributed objects are accessed transparently. If a client requests a service, it is either sent directly to the service object, if it is local, or to the (local) object reference ("proxy"), if it is remote. Calls on proxies are automatically intercepted by the ORB and forwarded to the remote object. Results of the request returned to the client appear the same as results of local requests. A HPDST ORB can handle multiple requests of external calls. The calls are mapped to independent processes in the Smalltalk image. ${ }^{1}$

\section{Standard core and standard object services}

HPDST provides support for standard core and for standard object services. Standard core services include support for the Interface Definition Language (IDL), static and dynamic invocation interfaces, and queries to an Interface Repository. HPDST provides a language binding IDL-to-Smalltalk which is expected to become part of the CORBA 2.0 standard. Public interfaces are registered in the Interface Repository which can be dynamically accessed. It allows clients to determine how to make requests to distributed service objects at run time.

Standard object services are not defined in the 1.1. specification. Hewlett-Packard and SunSoft have jointly proposed an extension for object services which is implemented in HPDST. It consists of services for object persistency, properties, associations, naming and eventing.

\section{Distributed Application Architecture (DAA)}

In addition to CORBA services HPDST provides support for Hewlett-Packard's Distributed Application Architecture (DAA), an specification for building cooperative, distributed object-oriented systems. The DAA, which is built upon CORBA class libraries, has strongly influenced our application so that we want to describe briefly its main concepts here.

DAA Object life cycle policies specify standard ways for objects to implement creation, deletion, copying and moving. The containment, link, and data view services deal with relationships between objects. Containment establishes a hierarchical relationship between objects. A container can contain any kind of DAA application object which is a common desktop object like a text document, business chart or a folder. They are presented to the user as one single entity, for example as an icon on the desktop. A container may contain other containers. The resulting link structure is called a containment tree.

The standard HPDST user interface uses an office-building containment tree. In that, the root container is called a building which contains an arbitrary number of office containers. An office can in turn contain desks, file cabinets, folders, etc.

1. External calls are not mapped to autonomous threads of the operating system. One reason is the virtual machine of the VisualWorks systems which does not support multi-threading at operating system level. 
HPDST provides many operations on containers and containment trees. The most important ones deal with object location management and object life cycle support. A container can be searched to locate its children, sub-trees can be moved to other containers by preserving the sub-tree structure and so on.

Using the containment model in combination with the CORBA class libraries allows the containers to be on different machines. This is a very powerful concept for building distributed, cooperative applications. Containers of objects can easily be moved to containers located on other machines. Remote containers can be traversed to locate, add, remove or modify objects.

Another central concept of the DAA used in our application is the presentation/ semantic split, which splits each distributed object into two parts, a presentation and a semantic object. The semantic object can be compared to the model in the Model-View-Controller (MVC) concept, because it contains all the important information about the application domain. Multiple presentation objects may be connected to one semantic object to allow the users to have different view on the same, shared data. Both semantic and presentation object do not have to be on the same machine. When the shared data of a semantic object is changed, all presentation objects will be automatically updated. Semantic and presentation object communicate via IDL.

\section{CREATING DISTRIBUTED OBJECTS WITH HPDST}

The creation of distributed objects with HPDST may start with the specification of their interfaces. ${ }^{1}$ CORBA distinguishes strictly between the implementation and the interface of an object. The interface must be defined in the programming neutral Interface Definition Language (IDL) while the implementation can be made in any programming language.

\section{Defining an object's interface}

To allow local Smalltalk objects to communicate with remote objects via the $\mathrm{ORB}$, the language neutral Interface Definition Language must be translated into a Smalltalk equivalent. The current CORBA 1.1 specification defines such a language binding for the $\mathrm{C}$ programming language only. HP's proposal for the Smalltalk binding used in HPDST is submitted to the OSF for the CORBA 2.0 specification.

IDL is a statically typed definition language. This makes a definition of a mapping from IDL to a dynamically typed language such as Smalltalk more difficult than for $\mathrm{C}++$, for example.

Simple IDL data types are mapped to the corresponding Smalltalk objects in category Magnitude (IDL long integer, IDL short integer $\rightarrow$ Integer, IDL float $\rightarrow$ Float, etc.). ${ }^{2}$ Smalltalk strings are mapped to IDL template type string. Because

1. Application development with HPDST does not necessarily have to start with the definition of the object interfaces. Alternatively, applications may be implemented by modifying existing (example) applications. IDL interfaces of existing Smalltalk classes might be defined later on.

2. In Smalltalk there is no notion of types. IDL types are all mapped to Smalltalk classes. 
Smalltalk class String consists of several subclasses (MacString, OS2String, ...) which would be mapped to all the same IDL type string, return string values can be specified with the pragma $\mathrm{CLASS}=<$ Smalltalk class name $>$. Using IDL pragmas allow the Programmer to use Smalltalk more flexibly in the sense that he is not restricted to the IDL data types. But the use of pragmas will make an interface definition implementation dependent, because other IDL compilers will probably not be able to interpret these directives correctly.

HPDST supports multiple inheritance of IDL interfaces. To avoid conflicts where an interface inherits from two interfaces of the same name but different implementations, IDL does not allow name overloading. Inherited interfaces can override types, constants and exceptions but not operations and attributes.

\section{Implementing the interface of an object}

The Smalltalk implementation of an object's interface can be made as usual by means of the VisualWorks development tools with two exceptions: First, to uniquely identify the interface and its implementation, each class must implement the instance method mostDerivedInterfaceID which returns the unique id of its interface. The system wide unique identifiers, can easily be obtained from the HPDST system. Second, each class must implement the abstractClassId method to allow the CORBA factory finder ${ }^{1}$ to uniquely identify the class to which instances can serve a request from a remote client.

\section{Creating and accessing objects in a remote image}

The way of accessing objects in a remote image depends on how the HPDST system is set up. According to the CORBA specification, HPDST can be used with at least one ORB and a unlimited numbers of Object Adapters (OA). To allow client objects to invoke methods of remote service objects, the server class must be registered in the server's factory finder. The interface of the server object must be registered in both the interface repository of the client and server image. A typical client invocation of a remote service looks like this (assuming a configuration with two ORBs running on remote machines):

1. get the naming service of the remote ORB: remoteNamingService := ORBObject namingService: 'hera.gmd.de'.

2. get the name of the remote factory finder by means of using the remote ORB's naming service:

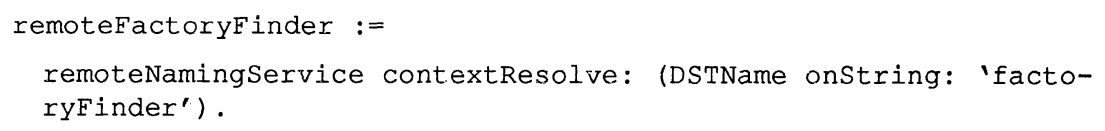

1. In CORBA terminology, an object which can be instantiated to create other objects is called a factory. Thus, all non-abstract Smalltalk classes can be used as factories. The factory finder is intended to find the appropriate factory whose instances can provide the service requested by an external client. The factory finder associates the object's abstract class id with its class name. 
3. create a new object with class name $\angle$ CLASSNAME $>$ on the remote machine using the abstract class id:

remoteObject:= remoteFactoryFinder createObject: <CLASSNAME> getInstanceACL.

4. send messages to the remote object as usual. The arguments in <MESSAGE $>$ must conform to the arguments of its IDL:

remoteObject <MESSAGE>.

In 4 . the call to the remote object looks exactly the same as a call to a local object. For the programmer there is no difference between a local and a remote object even if the remote object is created on a different platform.

\section{IMPLEMENTATION OF THE EVALUATION APPLICATION}

This section is concerned with the implementation details of our circulation folder application. It describes the basic concepts of the implementation and how we have used the concepts described above.

\section{Architecture overview}

The basic architecture of the circulation folder consists of a server and an unlimited number of clients (Fig. 4). Each client and the server are running Object

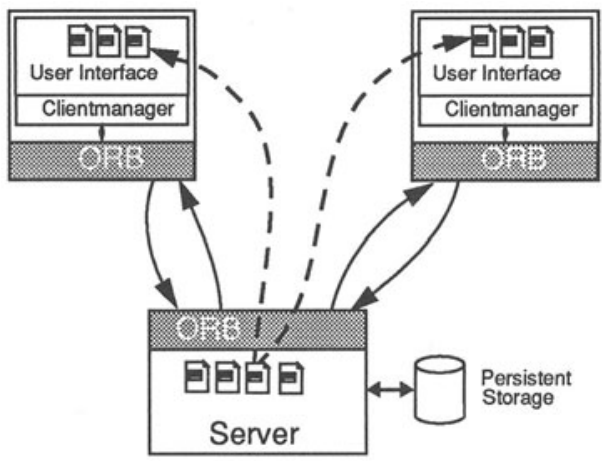

Fig. 4 Architecture of circulation folder application

Request Brokers in their Smalltalk images. In contrast to the classical client server architecture, the server can send messages to the currently connected clients at any time. Circulation folders are passed as physical copies from one client to another via the server. The server is responsible for the distribution of folders and manages a persistence storage of all circulation folders which can be used for recovery purposes after an system crash. The server keeps track of all currently connected clients. The solid arrows indicate method invocations from the clients to the server (e.g. send folder, get folder). The dotted arrows visualize the server-client communication which is invoked if the state (e.g. the position) of an circula- 
tion folder object changes. This concept allows a user to be aware of the current state of his circulation folders at any time.

In order to minimize network traffic and to enhance client autonomy we decided to pass circulation folders as physical copies. This allows a user to use a client without being connected to the server, e.g. on a mobile system. Eventing and information services about incoming circulation folders are handled by using CORBA mechanisms when the user reconnects to the server.

\section{Implementation details}

The current implementation makes intensive use of the DAA framework in combination with the HPDST CORBA class libraries.

In DAA terminology a circulation folder is simply a specialization of the DAA container classes. Since DAAContainer consists of a semantic and a presentation part (cf. Section 3) we subclassed both DAAContainerSO, and DAAContainerPO respectively. The contents of the control tag and the contents of the circulation folder itself belong to the view independent domain of the object. They are stored in the objects' semantic part. The presentation part of the circulation folder consists mainly of an adjustment of the views and the drag-and-drop protocol which allows a user to simply drag objects to and from the folder.

The implementation was straightforward. Almost all aspects concerning communication between the (distributed) parts of the objects were inherited from the superclasses. Our work was to define the IDL between both parts of the object, and to specify the persistent object data which have to be transmitted when the object migrates to a remote image.

The task of the client manager (Fig. 4) includes connecting and disconnecting to and from the server, requesting new circulation folders since a user's last login, transmitting folders to the server and propagating notifications about the progress of a particular folder to the user interface. The visual component of the client manager was shown in Fig. 1.

Connecting to the server is realized with the aid of CORBA's naming service (cf. also Section 4). The server coordinates all the circulation folders, passes them to the next user (in our terminology station), informs users about folders, that are currently in their in-boxes and actively notifies all users on a control tag list about the progress of the corresponding circulation folder (eventing service). Because these services are public to all (remote) clients, they have to be registered within CORBA's interface repository. HPDST's IDL differs in some points from standard IDL, but can be converted to standard syntax automatically. The most notable differences are the two pragmas CLASS, which were explained in Section 4, and SELECTOR. The second pragma should be added after every signature in an interface and specifies a mapping to a Smalltalk instance method.

Internally the server stores all circulation folders in a global dictionary keyed by a unique index. Actually, only small surrogates ("proxies") are kept in memory and point to regular files. The files serve as a persistency store. This workaround was necessary, because the persistent storage manager is still subject to OMG's standardization efforts and thus not yet available in the HPDST environment. 


\section{SOFTWARE DEVELOPMENT WITH HPDST}

This section describes our experiences with CORBA based software development under HPDST.

The use of CORBA technology has strongly affected many design and implementation decisions. CORBA allows implementation of the communication between objects at a high level. The low level protocol layer is completely encapsulated in Smalltalk classes. No work was necessary concerning data type conversion, serializing/de-serializing of objects, socket binding, etc. This encapsulation allowed us to design the whole application including communication layer fully in an objectoriented fashion independent of the platform.

However, using IDL as the interface language for Smalltalk objects restricts Smalltalk's flexible data type concept. Even simple Smalltalk data structures (e.g. Point, which contains two members each of arbitrary type) have to be awkwardly declared in IDL. Instances of more complex classes (e.g. dictionaries) can only be used in IDL if they are mapped by the programmer to IDL data types.

IDL is intended for definition of interfaces between objects which may not be implemented in the same programming language. However, IDL cannot cover the entire range of data types supported by all programming languages, so that there must be compromises in each particular IDL language binding. This means using IDL in a distributed Smalltalk application is reasonable when Smalltalk objects have to communicate with objects implemented in another programming language. However, there are currently no commercial ORB's available which support use of different implementation languages simultaneously. This is mainly due to the fact that the protocol between two ORB's is not yet defined (It's a major topic for the CORBA 2.0 standard).

Our implementation was simplified by the additional features supplied by HPDST, over and above those defined in CORBA 1.1. Many of these features, such as the naming service which provide a convenient way of locating remote objects, have been proposed as extensions to OMG and will probably be included with the CORBA 2.0 standard currently being defined.

On the other hand, there are many features still missing in CORBA 1.1 and HPDST. There are no means for implementing authentication, security and access rights which forced us to implement our own application dependent protection mechanisms. Some support for security is needed if CORBA is going to be used as the basis for industrial-grade applications.

As mentioned above, there is no object persistency store in HPDST. To make the circulation folders persistent we simply created a BOSS $^{1}$ stream on an object by calling the appropriate HPDST method. This stream wrote and read object details on the file system.

Again, this was a workaround not suitable for industrial applications. The use of a central database systems as an object store fails since it contradicts to the concept of distributed objects in the CORBA specification. The definition of a CORBA persistency store is an important future requirement.

The most powerful concept in HPDST for building the circulation folder applica-

1. Binary Object Streaming Service as provided in the VisualWorks System. 
tion was the Distributed Application Architecture which in turn uses CORBA distribution mechanisms. Distributed objects can easily be created by simply subclassing pre-defined DAA classes. These classes are tailored for groupware applications. Particularly the concept of the semantic/presentation split strongly simplified our implementation. By using the presentation and semantic split, we were able to cleanly separate the user interface components from the underlying application components. This allows for tailoring of the interface in isolation by both developers and users. This separation is important for groupware development, as cooperating users may work better with interface representations they can tailor individually [1]. Moreover, it allows reuse of the interface and application independently, and to easily the porting of the application to other platforms.

Outside the scope of CORBA but very useful in the development cycle was the HPDST remote debugger. It allows transparent inspection and debugging of objects which reside on remote machines. It allows access of objects in remote images as well as easy testing of the marshalling and unmarshalling process.

The HPDST application framework provides very short edit-compile-debug cycles even for distributed applications. The main reasons for this are the use of VisualWorks for the programming environment and the powerful concepts of the Distributed Application Framework which is based on the CORBA class libraries. The development of the evaluation application was finished in approximately 2 months with a team of 4 developers. Compared to our experiences with the development of similar applications, this is an enormous enhancement of productivity.

The performance of the evaluation application was sufficient ${ }^{1}$. A circulation folder containing up to 10 small-sized objects was transferred from one client to another almost immediately. The performance was dependent on the performance of the network instead on the performance on the application itself. This holds for many groupware applications.

\section{CONCLUSION}

We have shown how the use of the CORBA compliant class libraries and the Distributed Application Architecture (DAA) has simplified the design and implementation of the evaluation application. The use of CORBA and the DAA has led to a fully object-oriented design of the application which allows us to reuse parts of the application independently. In the development process we were able to separately design the user interface, the application, and the network layer.

However, CORBA 1.1 lacks important features. There is no way to connect ORB of different vendors since the protocol between ORB is not yet defined. This makes the use of different implementation languages currently impossible, although this is a major goal of OMG. Further requirements from a groupware developer point of view are the definition of object dependent standards concerning security, authentication and access rights.

HPDST provides support for concepts that are not defined in CORBA 1.1 but are submitted to OMG to be included in the 2.0 standard. These are the object naming

1. We used a cluster of Sun workstations. The server ran on a Sparc Station 10, the clients on Sparc IPX. 
service, a specification for information insertion into the repository (e.g. compiling IDL), a concept of asynchronous messaging (event channels), the IDL-to-Smalltalk language binding, and others. Most of them are advantageously used in our application.

We found HPDST is very powerful tool for building distributed, cooperative applications across different platforms. The network layer is completely encapsulated in Smalltalk classes. Together with the encapsulation of the operating system dependencies by the VisualWorks system, HPDST allows development of distributed applications which run without modification to the source or binary code on all major platforms. Due to the incremental compilation in the VisualWorks system, HPDST allows to rapid prototype distributed applications which strongly increases the productivity of system development.

\section{ACKNOWLEDGEMENTS}

The evaluation application was developed and implemented by Ulrich Frank, Rüdiger Grey, and the authors. Richard Bentley and Uwe Busbach read numerous drafts of the paper and made valuable comments.

\section{REFERENCES}

[1] Bentley, R., Rodden, T., Sawyer, P., and Sommerville, I. “Architectural Support for Cooperative Multiuser Interfaces”, IEEE Computer, Vol. 27, No. 5, May 1994, pp. 37-46.

[2] Grudin, J. "Why CSCW systems fail: problems in the design and evaluation of organizational interfaces" In Proc. CSCW'88, Portland, Sept. 1988, pp. 85-93.

[3] Grudin, J. "Groupware and cooperative work: Problems and prospects" In: Laurel, B. (Ed.): The art of Human-Computer Interface Design, Addison Wesley, 1990, pp. 171-185.

[4] Hoschka, P., Kreifelts, T., and Prinz, W. "Gruppenkoordination und Vorgangsbearbeitung" In: Kirn, S., and Klöckner, K. (Eds.): Betrieblicher Einsatz von CSCW-Systemen (in German), GMDStudien Nr. 230, St. Augustin, 1994, pp. 91-112.

[5] Kreifelts, T., Hinrichs, E., Klein, K.-H., Seuffert, P., and Woetzel, G. "Experiences with the DOMINO office procedure system" In: Bannon, L., Robinson, M., and Schmidt, K.(Eds): Proc. of the Second European Conference on Computer Supported Work-ESCW'93, Kluver, Dordrecht, 1991, pp. 117-130.

[6] Kreifelts, T., Hinrichs, E., and Woetzel, G. "Sharing To-Do Lists with a Distributed Task Manager" In: De Michelis, G., Schmidt, K., and Simone, C. (Eds.): Proc. of the Third European Conference on Computer Supported Work-ESCW'93, Kluver, Dordrecht, 1993, pp. 31-46

[7] Object Management Group "The Common Object Request Broker: Architecture and Specification", Revision 1.1, OMG Press, 1991.

[8] Prinz, W. "Providing organizational information to CSCW applications" In: De Michelis, G., Schmidt, K., and Simone, C. (Eds.): Proc. of the Third European Conference on Computer Supported Work-ESCW'93, Kluver, Dordrecht, 1993, pp. 139-154. 